\title{
Diseño de un sistema de automatización para las bahías de la subestación de San Bernardino
}

Fernando Martínez Santa*

Yamit Ricardo Lugo Sierra*

\section{Resumen}

Este artículo pretende mostrar el diseño del Sistema de Automatización de las bahías Jamondino 1, Jamondino 2 y Yumbo, de la Subestación de San Bernardino, ubicada en Popayán, departamento de Cauca. La subestación sirve como conexión al Sistema Interconectado Nacional, y surge como estrategia para mejorar el mantenimiento de los Sistemas de Automatización de Subestaciones, sAS. El artículo incluye el diseño y pruebas del sas de San Bernardino, que les permiten al operador tener todas las señales concentradas en un solo sitio, con el propósito de ejecutar sus acciones operativas de una manera más segura. De esa forma, el operador cuenta con la información necesaria en el tiempo oportuno, para impedir cometer errores en la operación de la subestación e incluso agilizar la reposición de los circuitos ante eventos imprevistos. También ofrece mayor facilidad para realizar mantenimiento y ampliaciones en el sistema, sin incurrir en altos gastos operativos.

Palabras clave: automatización, mantenimiento, ampliación, subestación, actualización, seguridad.

\begin{abstract}
This article aims to show the design of the Automation System of the Jamondino 1, Jamondino 2, and Yumbo bays of the San Bernardino Substation, located in Popayán, department of Cauca. The substation serves as a connection to the National Interconnected System, which arises as a strategy to improve the maintenance of Substation Automation Systems, SAS. The article includes the design and tests of the SAS of San Bernardino, which allows the operator to have all the signals concentrated in one place, in order to carry out their operational actions in a safer way, providing the necessary information in the opportune time to avoid making mistakes in the operation of the substation, and even speed up the replacement of circuits in the face of unforeseen events. This system makes it easier for the operator to perform maintenance and upgrades on the system without incurring high operating expenses.
\end{abstract}

Keywords: Automation, Maintenance, Expansion, Substation, Improvement, Security

* Profesor asistente Tecnología en Electricidad, Universidad Distrital Francisco José de Caldas. Contacto: fmartinezs@ udistrital.edu.co

** Ingeniero en Distribución y Redes Eléctricas, Universidad Distrital Francisco José de Caldas. Contacto: yrlugos@correo. udistrital.edu.co 


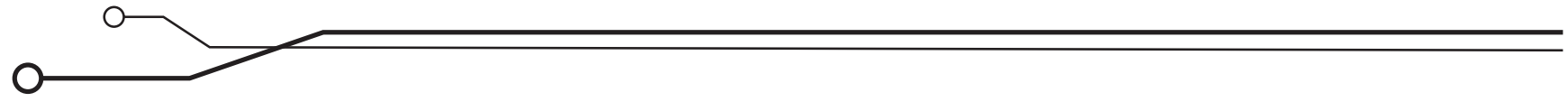

\section{Introducción}

Desde el principio de la existencia de las Subestaciones Eléctricas (SE) ha sido necesario disponer de sistemas de protección para las personas, equipos e instalaciones. Dichos sistemas de protección se encargan de mitigar cualquier posible condición anormal de trabajo, que pueda repercutir en daños para el sistema (generadores, transformadores, interruptores, líneas de potencia y barras).

Figura 1. Subestación eléctrica de San Bernandino.

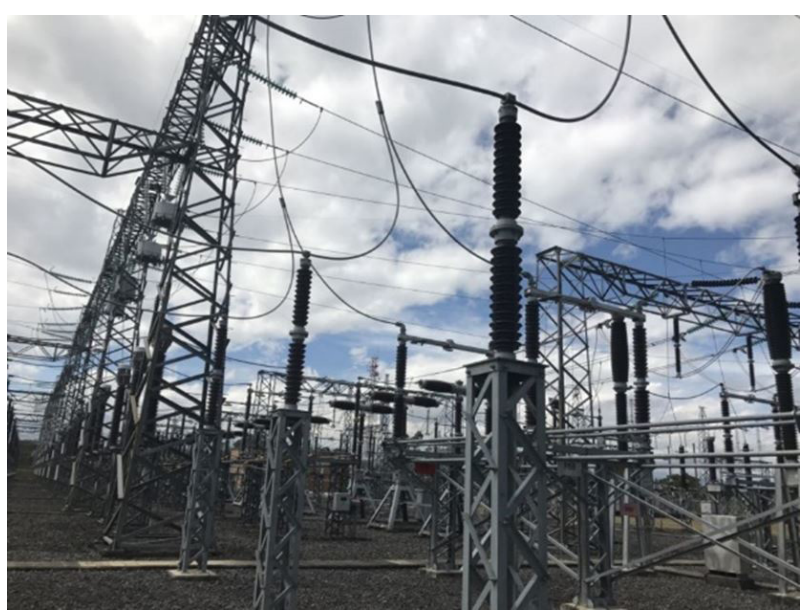

Fuente: elaboración propia.

Al principio, el trabajo de control y operación de las instalaciones era realizado por personal humano presente en las subestaciones (operadores de instalación). A medida que evolucionaron las redes eléctricas, también creció su tamaño y complejidad, lo que requirió de mayor supervisión y gestión de las instalaciones. La información suministrada en caso de actuación por las protecciones electromecánicas es muy limitada: usualmente se presenta un "testigo" de color rojo a nivel de protección, así como la actuación de contactos eléctricos de alarma, que avisan de la incidencia.

En la actualidad, el proceso de automatización de las SE es un proceso enfocado principalmente a la correcta operación y funcionalidad de los equipos que conforman la subestación eléctrica. Como se sabe (Chuncho, 2013), en la actualidad las subestaciones poseen equipos que, en su mayoría, tienen una antigüedad de entre 10 y 20 años, por lo que la integración de estos equipos en un sistema Scada, Supervisory Control And Data Acquisition (Supervisión, Control y Adquisición de Datos), se ve obstaculizada por su antigüedad. Este mismo problema también puede ocurrir en los niveles inferiores de tensión.

La eficiencia en la generación, transmisión y distribución de energía eléctrica debe, con bajos costos de mantenimiento, mejorar la competencia de las compañías en el mercado energético, para que así la SE permanezca disponible el mayor tiempo posible. También, deben realizarse estudios sobre gestión para proveer energía eléctrica, teniendo una SE en excelentes condiciones de funcionamiento, cuya funcionalidad esté basada en los estándares y, a su vez, cuente con 
la tecnología necesaria: si por algún motivo se presenta algún evento que pueda causar daños a personas o los equipos, esta tecnología pueda actuar de acuerdo dicho evento. Por estas razones, los diseñadores de SE deben estar a la vanguardia y apropiarse de las nuevas tecnologías, en cuanto a dispositivos que optimicen el desempeño del sistema eléctrico.

Para mejorar la funcionalidad de una SE, actualmente se debe tener en cuenta lo siguiente:

- Debe tener la capacidad de realizar control, gestión y operación de las variables eléctricas.

\section{Metodología}

Se envió un técnico a la subestación de San Bernardino, para hacer el levantamiento de señales y de información técnica para el desarrollo del proyecto: planos eléctricos, listado de equipos, dimensión de tableros y recopilación de señales de la subestación.

Los listados de señales que se trajeron de las tres bahías de la subestación de San Bernardino, con el respectivo posicionamiento en los PLC, Programmable Logic Controller (Controlador Lógico Programable), y borneras. La arquitectura general se inició con la cantidad de señales de entrada y de salida que se recopilaron en la subestación.

A continuación, se explica la configuración del PLC Quantum. Se utilizaron 71 entradas digitales. Para esta cantidad, se usaron tres tarjetas de entradas digitales 140ERT85410 (Schneider electric $\AA$, 2011), con 32 señales disponibles por

\section{PLC Twido}

El cliente solicitó una redundancia en las señales de apertura y cierre de los equipos de cada bahía, para lo que se utilizó un PLC Twido
- Debe ser monitoreada y gestionada desde un sitio remoto, por medio de enlaces de comunicación estándar.

- Debe tener un sistema de control en tiempo real para ejecutar las estrategias de protecciones y maniobra.

- Debe usar varios sensores para recolectar la información de la SE.

- Debe tener mejoras basadas en estándares y regulaciones internacionales.

tarjeta, lo que dejó a la última tarjeta con 25 señales de reserva, para cuando la empresa ISA S.A. desee agregar más señales al sistema.

Se usaron 11 salidas digitales. Para esta cantidad, se utilizó una tarjeta de salidas digitales 140DRA84000, con 18 señales disponibles por tarjeta, lo que dejó 5 señales de reserva, para cuando la empresa ISA S.A. desee agregar más señales al sistema.

También se utilizó una CPU con referencia 140CPU31110, una tarjeta de comunicación 140NOE77101 y una fuente 140CPS52400, para la alimentación del backplane, cuyas características técnicas se muestran en el Anexo B. Se utilizó un backplane de 10 casillas (slots). Teniendo en cuenta que la cantidad de tarjetas ocupó 6 casillas, se dejó una reserva de 4 casillas. para una ampliación del sistema.

(Telemecanique, 2007a), de referencia TWDLCDA24DRF, con 14 entradas digitales y 10 señales de salidas digitales. Las señales de entradas 


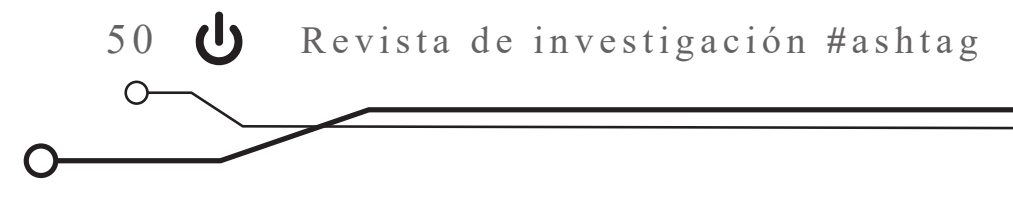

digitales mínimas para realizar la redundancia fueron 15, por lo que se agregó un módulo de entradas digitales TWDDDI8DT, que dejó como reserva 7 entradas digitales.

Se utilizó una IHM, Interfaz Hombre-Máquina, con referencia Magelis XBTGT2110 (Telemecanique, 2007b), que se incluyó dentro de nuestra

\section{Características de los tableros}

Los tableros fueron de tipo auto soportado, con ensambles metálicos, diseñados y construidos de acuerdo a los tableros de controles y sistemas industriales. Se instalaron protecciones IP54 para ambientes contaminados y alta humedad. Los ensambles metálicos utilizados fueron rígidos, reforzados, libres de abolladuras, rayones, huecos, defectos en general, e indeformables por el peso propio de los equipos. Los bordes fueron laminados, de manera que formaran un ángulo, y las esquinas se soldaron y se pulieron suavemente. El color de acabado es gris claro RAL 7032.

Se definió la disposición de los cubículos y se tuvo en cuenta el espacio suficiente para la ubicación del medidor multifuncional, de control y controlador PLC principal y de respaldo; interfaz humano máquina, selectores, interruptores y bornas de control, de tal manera que hubiera una buena distribución en el tablero. Las conexiones de los interruptores derivados a las barras fueron atornilladas de tal forma que se sujetaran firmemente a las barras, bajo condiciones de alta corriente de falla.

El tablero y los paneles o cubículos tienen una barra de cobre, para conectar a tierra los equipos; y el marco del tablero tiene una sección suficiente para conducir la corriente de cortocircuito máxima. A cada barra de puesta a tierra oferta, para el manejo del sistema desde la sala de control.

También se incluyeron relés repetidores de señal, bornas seccionables, con sus respectivos accesorios; y bornas cortocircuitables, para el medidor multifuncional, proporcionado por el cliente.

se le suministraron terminales, para conectar los cables exteriores de cobre.

Se dispuso de puertas con bisagras internas en la parte frontal del tablero. Cada puerta tuvo una cerradura con pestillos en la parte superior, media e inferior, accionadas por un mismo mecanismo provisto de llave. Las puertas en la parte interior del marco tuvieron empaquetadura de neopreno. Las dimensiones del tablero en ningún caso fueron diferentes a las mencionadas a continuación: $2.2 \mathrm{~m}$ de alto, $0.8 \mathrm{~m}$ de ancho, $0.8 \mathrm{~m}$ de profundo y $0.5 \mathrm{~m}$ de base (ver figura 2 ).

Figura 2. Tablero de control de bahía.

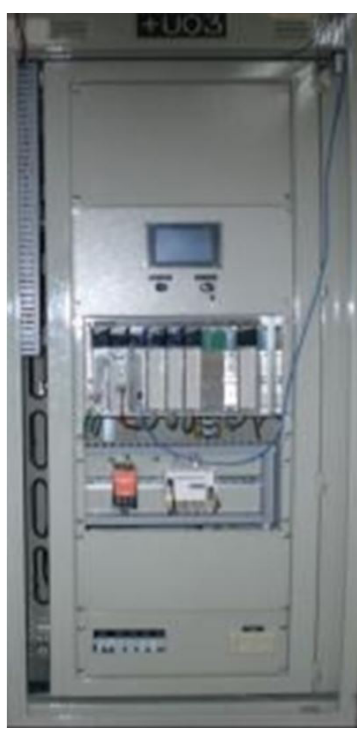

Fuente: elaboración propia. 
Dentro del tablero se suministró un calentador de espacio que se instaló en la parte más baja del tablero, y se ubicó de tal manera que permitiera la circulación del aire y evitara sobrecalentamientos. El control del calentador se calibró de acuerdo a la temperatura y humedad relativa.

\section{Manejo de software AutoCAD®, Eplan®}

Para dar comienzo al diseño eléctrico y mecánico, se explica qué tipos de software se utilizaron para elaborar los planos eléctricos. En este diseño, se utilizó el software Eplan 5.0® y el software AutoCAD®, que, por sus herramientas y librerías de símbolos eléctricos, dan una destreza para el desarrollo de este diseño. El software Eplan 5.0® cuenta con un sistema de unión de puntos y referencias cruzadas para una misma conexión automáticamente, vinculando esquemas unifilares y trifilares. Además de esto, ofrece tablas de cableado para su conexionado, diagramas de conexión de borneras configurables y formatos de elaboración con designaciones de página, versión, cliente y códigos necesarios que pueden ser establecidos por el usuario.

\section{Memoria de cálculos}

Para realizar la requisición de equipos, se realizaron los siguientes cálculos. Se utilizó un breaker para servicios auxiliares lámpara, resistencia, tomacorriente, de 6A, referencia 24399, fabricante Merlín Gerin® -QAC1.

$$
P=V^{*} I
$$

(Ecuación 1)
Para la parte de servicios auxiliares se agregó un bombillo de 60W a $120 \mathrm{VAC}$, interruptor de puerta microswitch; un tomacorriente monofásico doble de dos polos y tres hilos para 6 A, 120 VAC con terminal de tierra. Los cables de control dentro del tablero se llevaron por canaletas con un buen comportamiento al fuego. En la zona superior del armario se instaló una placa con el nombre y la descripción del tablero.

En los planos eléctricos y mecánicos, se especificó el conexionado de control por medio de unión de líneas entre puntos de conexión, placa de nomenclatura, tipo de barraje y tipo de cable, que se usó para el alambrado. Esto sirve como guía para el ensamble en la codificación de cables y marquillas del tablero, características propias y referencias de los equipos utilizados en el diseño, entre otros.

El diseño inició dando un arreglo de la arquitectura general, que se reformó según las especificaciones técnicas mencionadas anteriormente, e involucró todos los elementos asociados a la realización del diseño del SAS de las bahías Jamondino 1, Jamondino 2 y Yumbo, de San Bernardino.

Los consumos de los elementos son: una resistencia de 100W, un bombillo de 60W, y un tomacorriente, que puede tener una carga de consumo de hasta 500W.

Por lo tanto: 


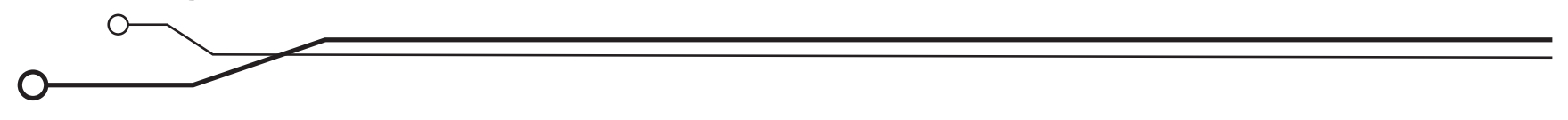

$$
\begin{gathered}
P=(100+60+500) \mathrm{W}, \text { entonces } \\
P=660 W I=\frac{P}{V} \\
I=\left(\frac{660}{110}\right) A \\
I=6 A
\end{gathered}
$$

(Ecuación 2)

También se utilizó un breaker bipolar para corriente continua tipo C32 H-DC, para la protección de los equipos con alimentación a 125VDC, fuente del PLC Quantum, tarjetas 140ERT85410, medidor multifuncional, entradas digitales a campo, fuente Weidmuller ${ }^{\circledR}$, de $10 \mathrm{~A}$, referencia 20545 fabricante Merlin Gerin ${ }^{\circledR}$ - QCC1 (ver ecuación 1).

Los consumos de los equipos son: alimentación externa en campo, protegida por un interruptor de 2A; fuente del PLC Quantum, medidor multifuncional y tres tarjetas 140ERT85410 (Schneider electric $\left({ }^{\circledR}, 2009\right)$ ), protegidas por un breaker de $3 A$, fuente Weidmuller ${ }^{\circledR}$ referencia 8708660000 , consumo máximo de 250W.

Por lo tanto:

$$
\begin{gathered}
P=(2 * 125)+(3 * 125)+250 \mathrm{~W}, \text { entonces } P=875 W \\
I=P / V \\
I=\left(\frac{875}{125}\right) A \\
I=7 A
\end{gathered}
$$

(Ecuación 3)

Después, se utilizó un breaker monopolar para corriente continua tipo C32 H-DC, para la protección de entradas digitales a campo a 125VDC
2A, referencia 20532 fabricante Merlín Gerin® -QCC2 (ver ecuación 1). Se definió que el consumo máximo que pueden tener estos equipos son de 250W. Por lo tanto:

$$
\begin{gathered}
P=250 \mathrm{~W} \\
I=P / V \\
I=\left(\frac{250}{125}\right) \mathrm{A} \\
I=2 \mathrm{~A}
\end{gathered}
$$

(Ecuación 4)

Luego se utilizó un breaker monopolar para corriente continua tipo C32 H-DC, para la protección de la fuente del PLC Quantum, tarjetas 140ERT85410, medidor multifuncional de 3A, referencia 20533, fabricante Merlín Gerin $®$-QCC3 (ver ecuación 1). Los consumos de los equipos son: fuente del PLC Quantum 140CPS52400, que tiene un consumo de $62.5 \mathrm{~W}$; tres tarjetas de entradas digitales 140ERT85410, que consumen 200W; y el medidor multifuncional, con un consumo de 56W. Por lo tanto:

$$
\begin{gathered}
P=(200+62,5+56) W, \text { Entonces } \\
P=319 W \\
I=P / V \\
I=\left(\frac{319}{125}\right) A \\
I=2,55 A \\
\text { (Ecuación 5) }
\end{gathered}
$$

Por último, se utilizó un breaker monopolar para corriente alterna tipo $\mathrm{C} 60 \mathrm{~N}$, para la protección de la fuente Weidmuller ${ }^{\circledR}$ de $4 \mathrm{~A}$, referencia 24398, fabricante Merlín Gerin® -QCC4 (ver ecuación 1). 
La carga máxima que puede soportar la fuente es de 3.9A, utilizando un breaker de corriente alterna, que tiene una curva de disparo más rápida que el de corriente continua. Estos breakers pueden reemplazarse por interruptores en corriente continua de máximo $60 \mathrm{~V}$.

En el mercado se consiguen breakers de las siguientes corrientes:

Figura 3. Listado comercial de interruptores

\begin{tabular}{|c|c|c|c|}
\hline Referencia & Tipo & In (A) & Poder de corte (KA) \\
\hline 24067 & C60N & 0.5 & 20 \\
\hline 24395 & C60N & 1 & 20 \\
\hline 24396 & C60N & 2 & 20 \\
\hline 24397 & C60N & 3 & 20 \\
\hline 24398 & C60N & 4 & 20 \\
\hline 24399 & C60N & 6 & 20 \\
\hline 24401 & C60N & 10 & 20 \\
\hline 24403 & C60N & 16 & 20 \\
\hline 24404 & C60N & 20 & 20 \\
\hline 24405 & C60N & 25 & 20 \\
\hline 24406 & C60N & 32 & 20 \\
\hline 24407 & C60N & 40 & 20 \\
\hline 24408 & C60N & 50 & 20 \\
\hline
\end{tabular}

Fuente: elaboración propia.

La curva de disparo C, de la marca Schneider electric $®$, línea Merlin Gerin $®$, fue la que se utilizó en este proyecto para todos los breakers, debido al tipo de trabajo que se realizó. A continuación, se da una breve descripción de esta curva, como lo muestra la figura 4.

Figura 4. Curva de disparo c.

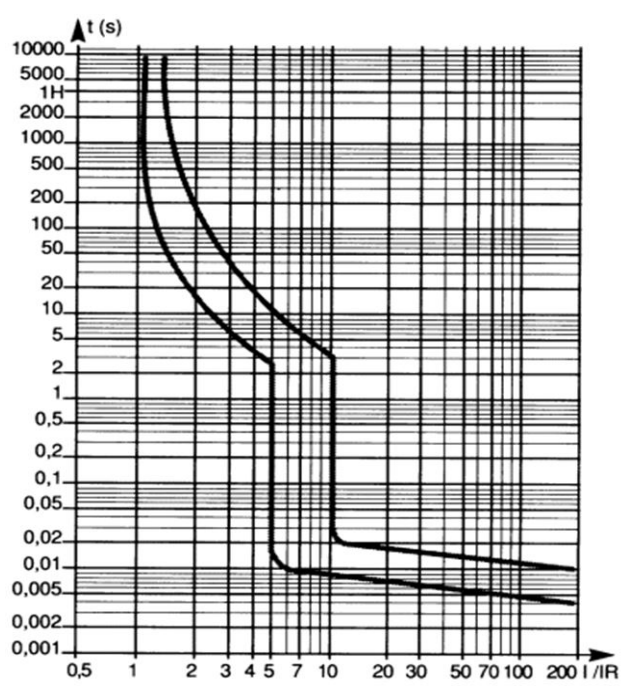

Fuente: elaboración propia. 


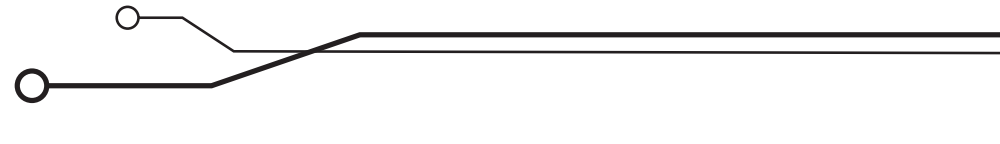

Estos magnetotérmicos actúan, en su zona térmica, entre 1,13 y 1,45 veces la intensidad nominal,; en su zona magnética, entre 5 In y 10 In; o en corriente continua, de 7 In y 10 In, según el tipo de aparato, de acuerdo con las normas IEC 60898 e IEC 60947-2 (Toscano, 2010; Salazar,
2015). Los magnetotérmicos se aplican para evitar los disparos intempestivos, en el caso de la protección de receptores que presentan, una vez en servicio, puntas de corriente de cierta consideración y se utilizan en las instalaciones de líneas-receptores.

\section{Programas en la Інм para el manejo de los equipos de las bahías Jamondino 1, Jamondino 2 y Yumbo.}

Para ingresar las señales al programa Vijeo Designer, se hizo el mapeo de los PLC Quantum y Twido de cada bahía.

\section{Implementación de señales en la pantalla táctil Magelis XBTGT-21 10}

Con el mapeo de señales de los PLC, se ingresaron las señales en las IHM de cada una de las bahías, de la siguiente manera. Primero, se instaló el software de la pantalla táctil Magelis XBTGT-2110 (Vijeo Designer). Luego, se ingresaron, a un ítem nombrado, variables en las que aparecían dos opciones, variable de tipo interno o externo, cada una de las cuales se podía subclasificar en señal discreta, flotante, entera o cadena. Para crear la variable se dio clic derecho sobre la clasificación, interno o externo, en la que aparecía una lista con las subclasificaciones, de la que se escogió la deseada. Lo siguiente que se realizó fue dar doble clic en la variable creada, como aparece en la figura 5. Allí se asignó el nombre, se le dio la subclasificación, se agregó la posición de memoria y, por último, se escogió el PLc, del cual se iba a traer la señal; todas las señales se crean de la misma forma. Para la programación de esta IHM, se utilizó el manual de la Magelis XBTGT 2110 (Telemecanique, 2007b).

Figura 5. Descripción de variable.

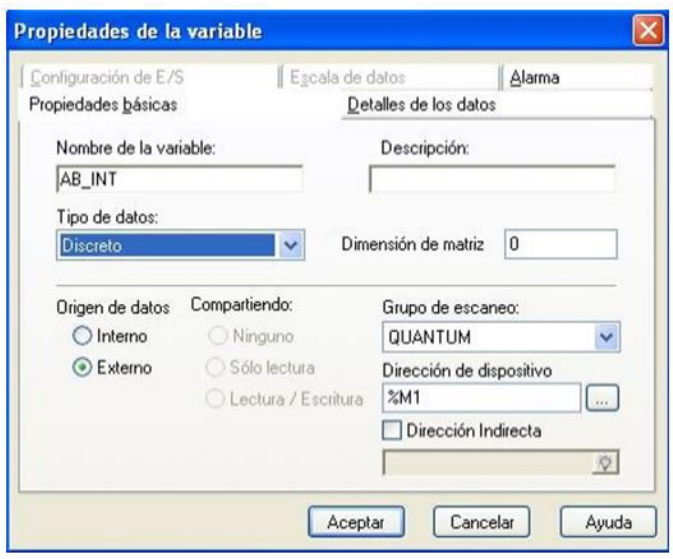

Fuente: elaboración propia. 


\section{Diagrama de flujo}

Para saber qué paneles se ingresaron en el programa y la secuencia de funcionamiento, se realizó un diagrama de flujo que se muestra en la figura 6.

Figuran 6. Diagrama de flujo general.

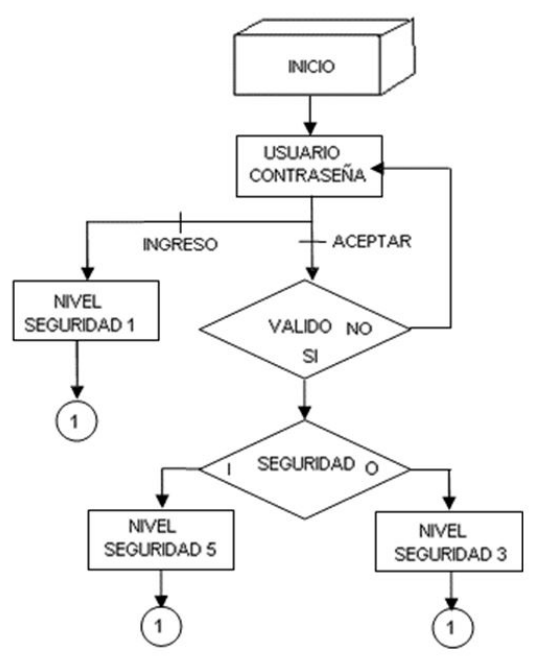

Fuente: elaboración propia.

Figura 7. Diagrama de flujo general.

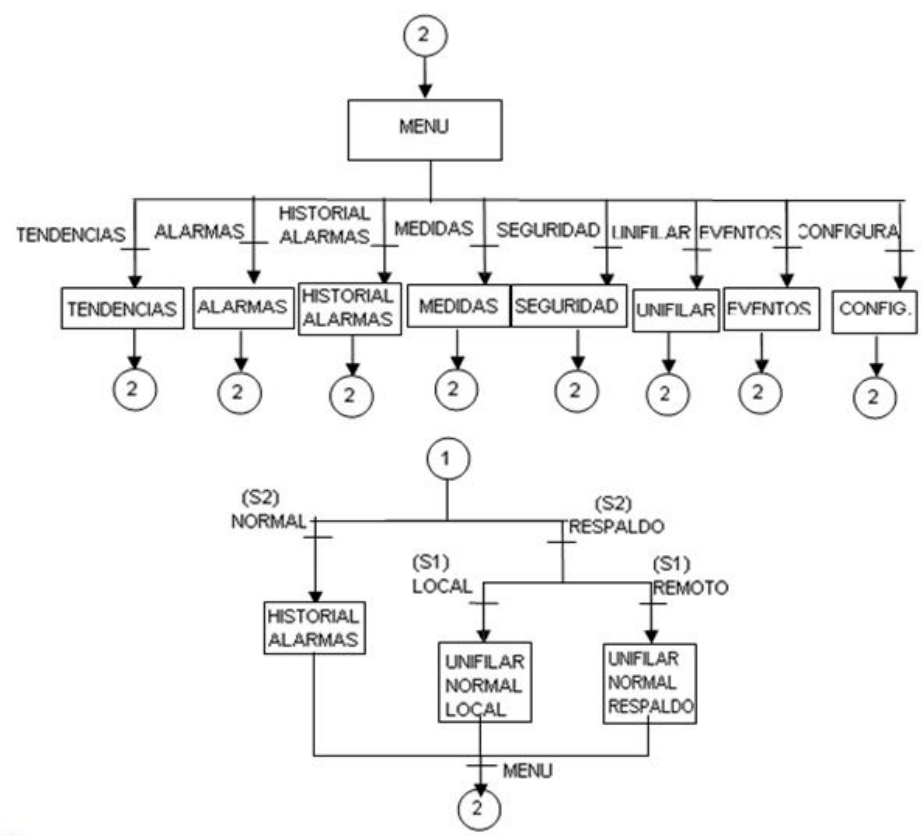

Fuente: elaboración propia. 


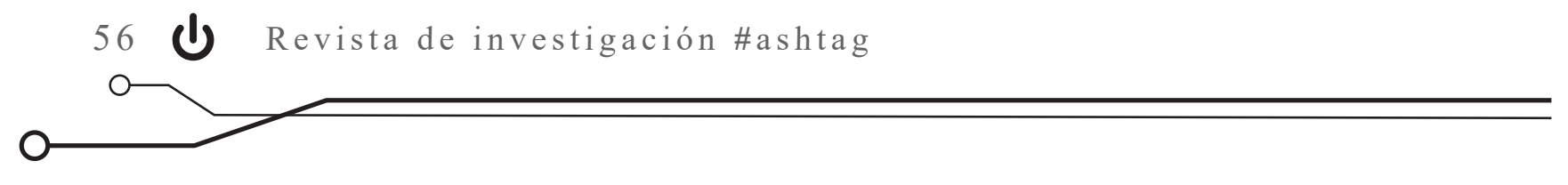

\section{Diseño de paneles e icono del programa}

Para realizar el diseño de los paneles e iconos que se utilizaron en el programa, se tuvo en cuenta el diagrama de flujo para crear los paneles principales y se asignaron los iconos que se

muestran en la explicación de la implementación del programa, en la que algunos de ellos se asignaron de la biblioteca del programa y otros de descargaron de Internet.

\section{Asociación de animaciones a sus respectivas variables}

Para asociar las animaciones a sus respectivas que tiene el software en su manejo. Una de las cavariables, se tuvo en cuenta los diagramas lógicos que se muestran en el ítem Programación, del PLC marca Quantum.

Las animaciones que se le dieron a los iconos fueracterísticas que se tuvieron en cuenta para que el icono desempeñara su trabajo fue la operación. De dichas operaciones, se utilizaron, entre otras, cambio de panel, panel emergente, alarma y sonido.

ron fáciles de asociar, dada la baja complejidad

\section{Aplicación del programa en la XBTGT2110 para la bahía JAMONDINO 1}

En este proyecto se va a utilizar una IHM de una programación por medio de animación. A marca Schneider Electric ${ }^{\circledR}$ Telemecanique, de continuación, se dará una breve explicación de la familia XBTGT2110. Este programa se basa en cómo utilizar el programa.

Figura 8. Panel de ingreso nivel 1.

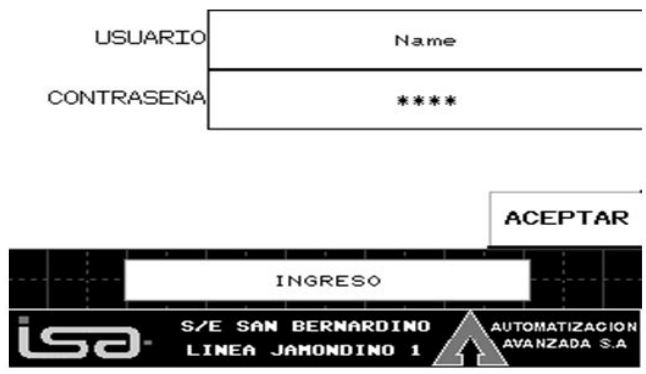

Fuente: elaboración propia.

Este panel tiene la función de iniciar la aplicación como usuario de operación o visualización según la operación (ver figura 8). 
Figura 9. Panel bahía controlador normal local nivel 1.

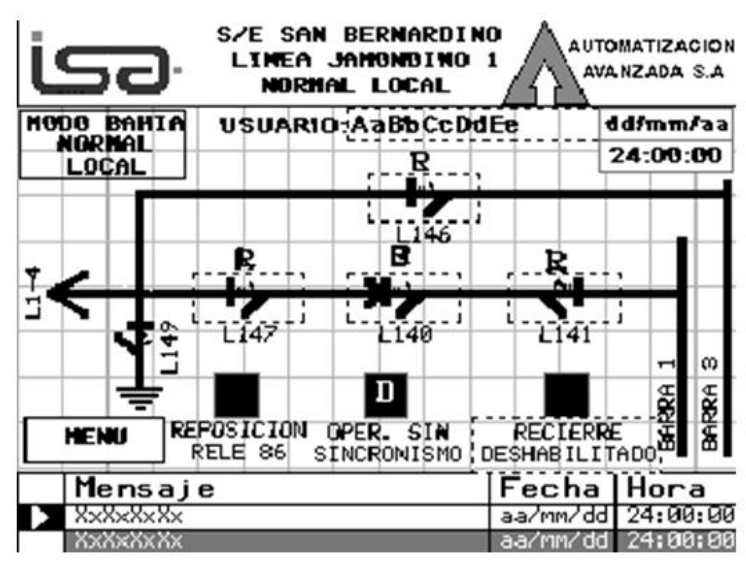

Fuente: elaboración propia.

En este panel (figura 9) se puede observar y dar ejecución a comandos dobles (Abrir o Cerrar), para los equipos de la bahía en modo Local y Normal, según enclavamientos de nivel
1, controlador principal. También se observan alarmas o disparos de los equipos de la bahía en modo normal, según listado de señales.

Figura 10. Panel bahía controlador respaldo nivel 1 .

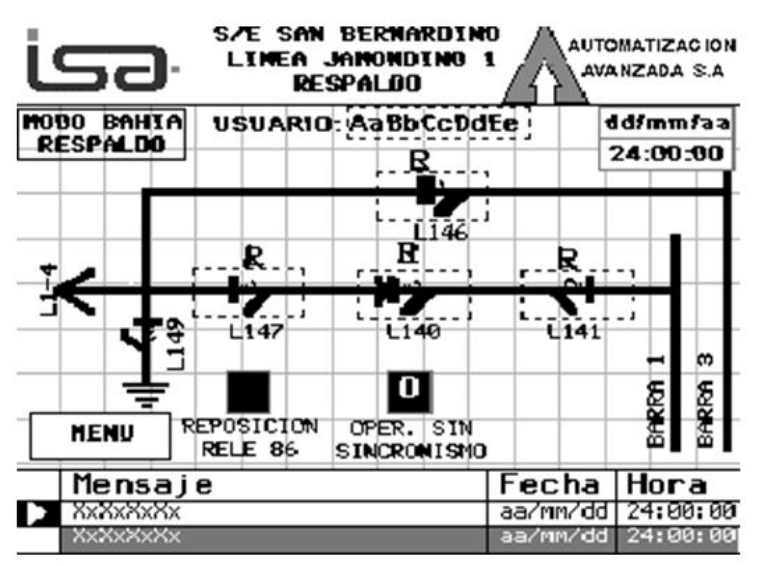

Fuente: elaboración propia.

En este panel se puede observar y dar ejecución a comandos dobles (Abrir o Cerrar), para los equipos de la bahía en modo respaldo, según enclavamientos de nivel 1, controlador respaldo. También se visualizan alarmas o disparos de los equipos de la bahía modo Respaldo. 


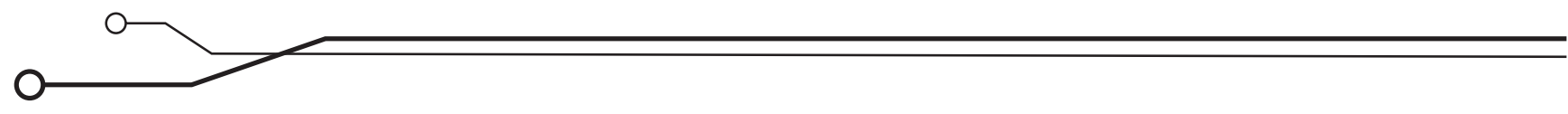

Figura 11. Panel de telemedidas.

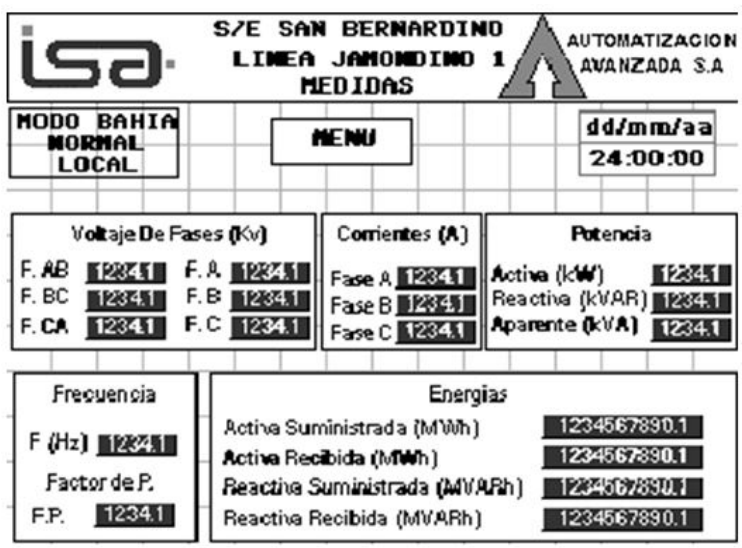

Fuente: elaboración propia.

En este panel se observan las telemediadas de tensión, corriente, potencia y energías de la línea con el controlador principal (Andreula, 2010)

(ver figura 11).

Figura 12. Panel de Historial de alarmas.

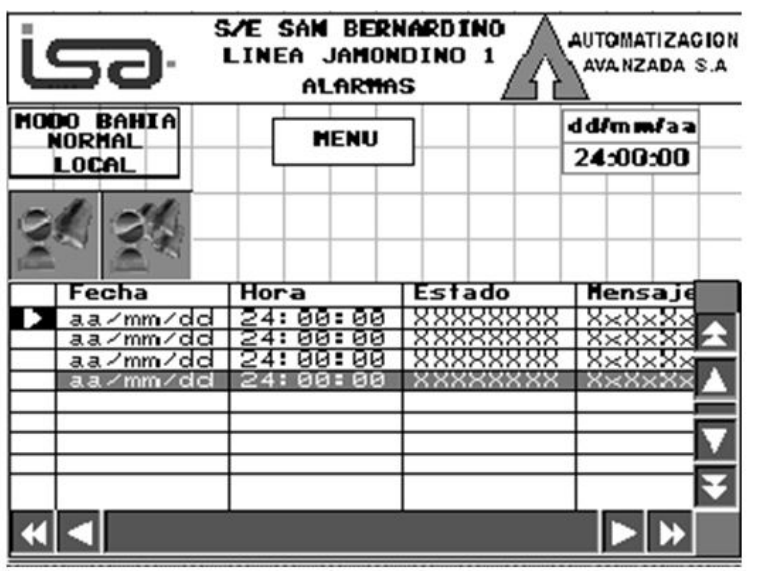

Fuente: elaboración propia.

En este panel se pueden observar las alarmas o disparos de los equipos de la bahía, según el listado de señales, teniendo en cuenta los respectivos modos de operación: Normal o Respaldo (ver figura 12). 
Figura 13. Panel de posición de los equipos.

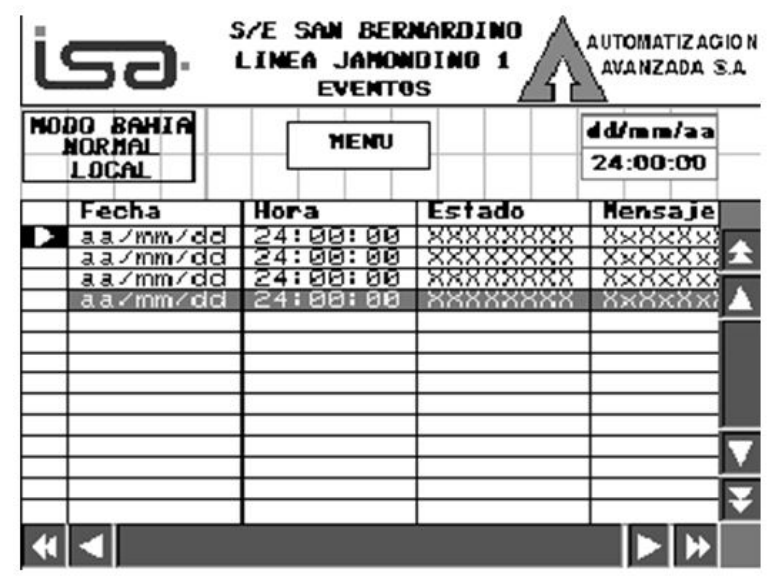

Fuente: elaboración propia.

En el panel de posición de equipos (ver figura 13), se pueden observar los eventos de posición para los equipos de la bahía, según el listado de señales, teniendo en cuenta los respectivos modos de operación: Normal o Respaldo. En la figura 14 se pueden observar las tendencias de voltaje, corriente, potencia activa y reactiva.

Figura 14. Panel de tendencias de variables.

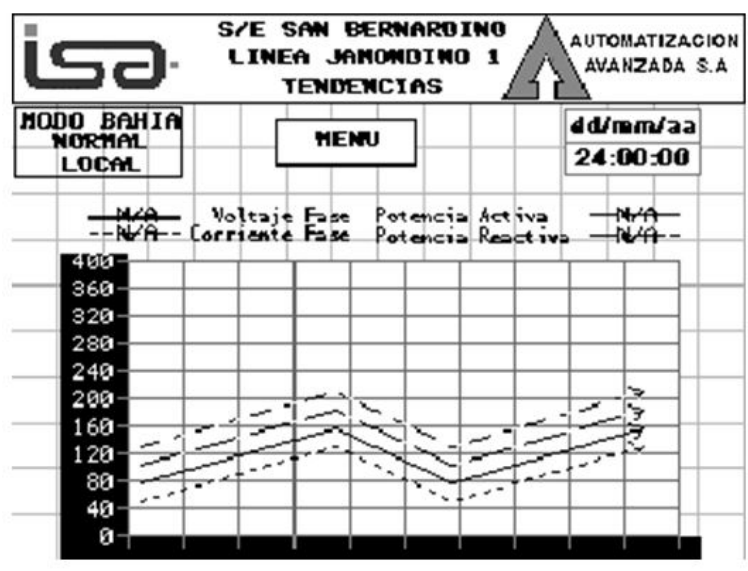

Fuente: elaboración propia.

En el panel de tendencias variables (ver figura 15), se puede agregar un usuario con su respectiva contraseña. También podemos definir y observar las variables internas del sistema de configuración de la IHM. 


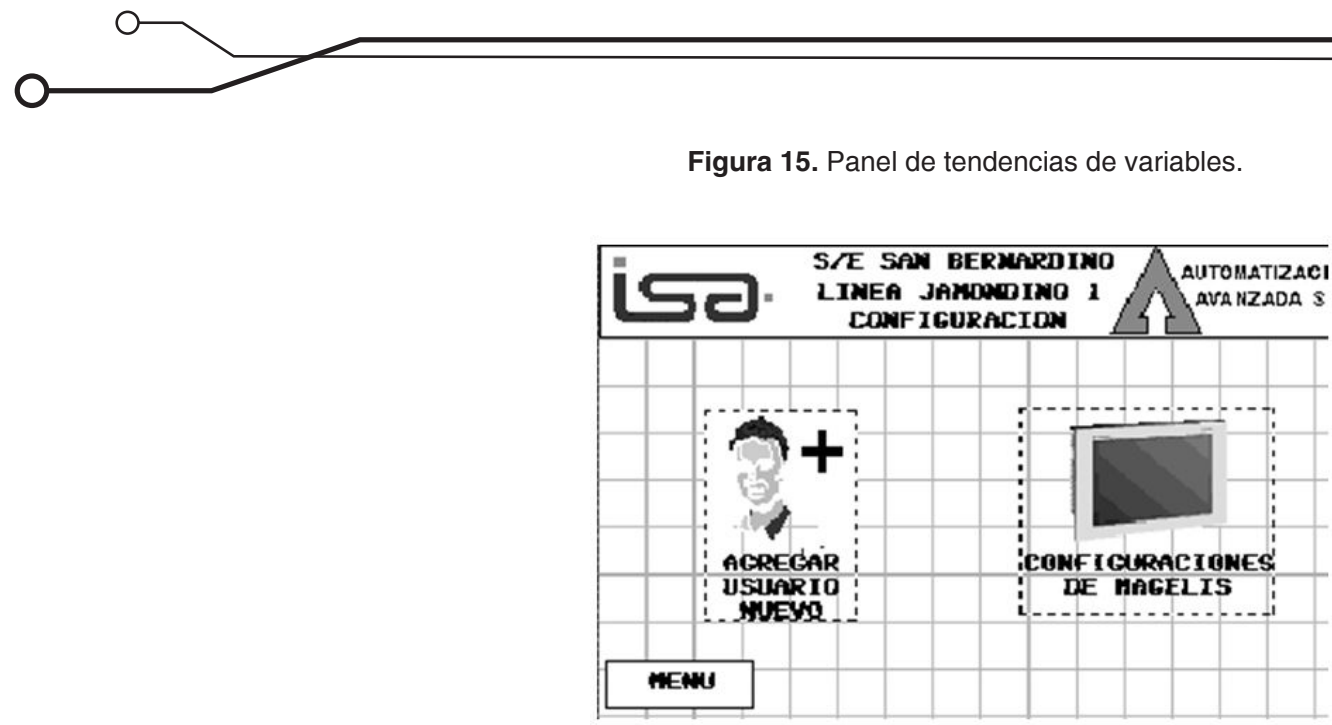

Fuente: elaboración propia.

En el panel de usuario de operación (ver figura 16), se ingresan los usuarios nuevos, al dar un nivel de seguridad al usuario. En este panel se encuentran campos como visualización, operación e ingeniería, y se le da la clave de usuario. El único que puede agregar usuarios es el usuario con nivel de ingeniería, ya que este tiene desactivados los bloqueos de los niveles inferiores.

Figura 16. Panel de usuario de operación.

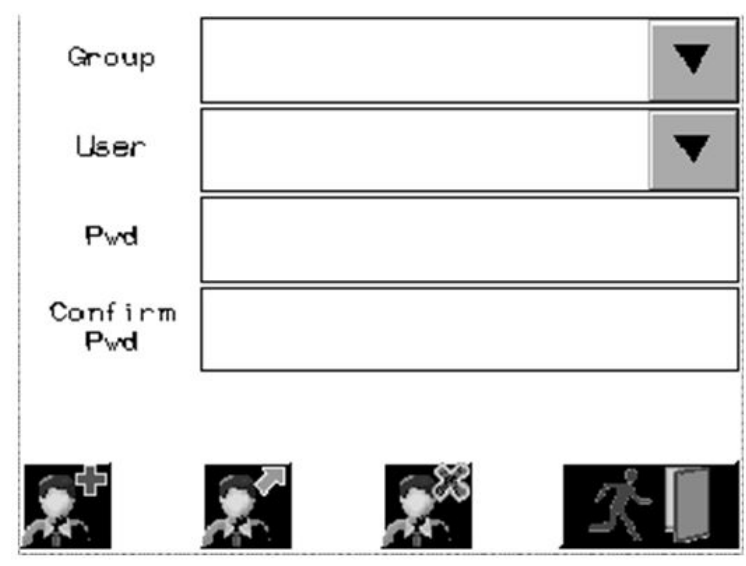

Fuente: elaboración propia.

Por último, se pueden encontrar todos los paneles que se mencionaron y explicaron anteriormente: dando clic en cualquiera de los iconos el programa, se abre la pantalla deseada (ver figura 17). 
Figura 17. Panel de tendencias de variables.

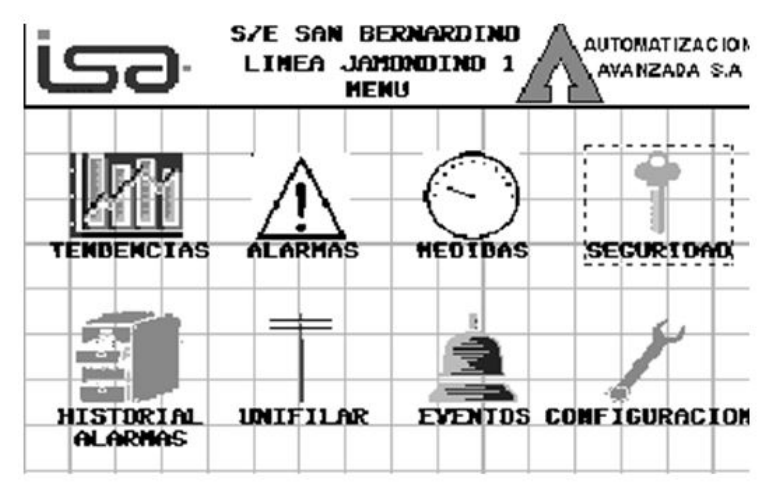

Fuente: elaboración propia.

\section{Programas de los PLC para el control de las bahías Jamondino 1 , Jamondino 2 Y Yumbo}

Se debe saber que el PLC Quantum es el PLC principal que ejecutará todo el programa y, en caso de cualquier falla en este equipo, entrará como respaldo el PLC Twido, que tendrá los comandos

\section{Programación del PLC marca QUANTUM}

Se instaló el software UnityproXL@, para iniciar la programación en el PLC Quantum. Se insertaron y se ubicaron las tarjetas en el backplane, lo que dejó tres casillas de reserva. También se utilizaron los manuales de cada tarjeta Quantum

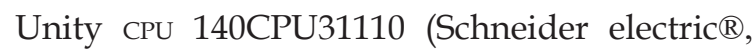
2009), y Modicon Quantum 140ERT85410 (Schneider electric $\left.{ }^{\circledR}, 2011\right)$.

Se abrió el programa UnityproXL $®$, en el que lo primero que se colocó fue la referencia de la CPU: 140CPU31110. Luego de esto, el programa abrió una ventana con un backplane de 16 casillas. Este backplane se cambió, dada la necesidad de un backplane de 10 casillas, que se incorporó al programa dando clic en el número 1, localizado al lado izquierdo del backplane. La referencia del bastidor que se asignó fue 140XBP01000. básicos para que el sistema no tenga un colapso. Pero primero, se dará la explicación del diseño del programa en el PLC principal o Quantum.

Después de tener esto, se empezaron a insertar las tarjetas que se designaron para el desarrollo del programa, dando doble clic sobre la casilla vacía, integrando así todas las tarjetas al sistema con su respectiva referencia. Se tuvieron presentes las señales que se recopilaron en cada una de las bahías de la subestación, para desarrollar los enclavamientos lógicos para cada equipo que compone la bahía.

Para realizar los enclavamientos lógicos, se tuvo en cuenta lo que el cliente requería. Después de tener los diagramas lógicos, se inició la programación de los PLC marca Quantum.

Antes de iniciar con la explicación del programa, se debe tener presente que, en las subestaciones y en procesos de ejecución especiales, se utilizan herramientas de alta gama, como lo son las 


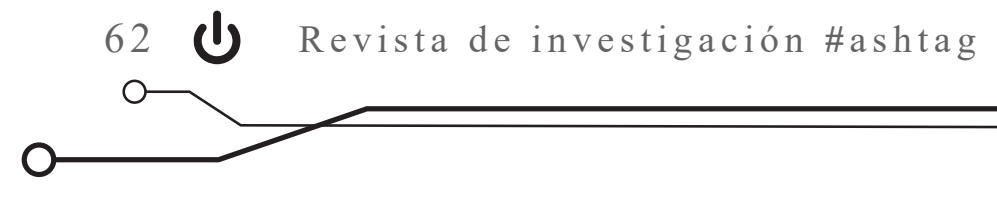

pantallas táctiles, Scada; y en procesos más complejos, la recopilación de señales desde un lugar a gran distancia, por ejemplo, las señales que son enviadas desde otra ciudad u otro país.

Los niveles que se utilizan en las subestaciones se describen a continuación:

- Nivel cero: accionamiento de equipos desde patio.

- Nivel uno: accionamiento de equipos desde un interfaz humano máquina.

- Nivel dos: accionamiento de equipos desde un Scada.

- Nivel tres: accionamiento de equipos desde el centro nacional de despacho.

En este proceso, se implementaron dos selectores de dos posiciones cada uno: el primero se encuentra etiquetado Normal, Respaldo; y el segundo selector se etiquetó con las posiciones Local, Remoto. Cuando el selector se encuentra en posición Normal, significa que el PLC que se encuentra realizando el trabajo es el de marca Quantum; cuando el selector se encuentra en

\section{Programación del PLC marca Twido}

La programación del PLC de respaldo se realizó con los enclavamientos más importantes del sistema. Teniendo en cuenta la cantidad de entradas y salidas que se podían incluir a este PLC, los pasos que se realizaron fueron muy parecidos a los pasos del PLC principal, entre ellos, la recopilación de señales en la subestación. Después de tener estos listados de señales, se tienen en cuenta los diagramas lógicos anteriormente mencionados. Luego, se inició con la programación del PLC, por medio del software Twidosoft 3.5 (Telemecanique, 2007a), utilizando el lenguaje Ladder. Con las posiciones de memoria se pueden realizar maniobras en el programa sin necesidad de que sean entradas físicas. modo Respaldo, significa que el PLC que está ejecutando el trabajo es el de marca Twido (Telemecanique, 2007). Cuando el segundo selector se encuentra en modo Local, significa que está ejecutando el trabajo desde la IHM; y cuando este selector se encuentra en modo Remoto, significa que el trabajo lo está realizando el Scada.

De acuerdo a lo que se estableció en los diagramas de enclavamientos lógicos, se procedió a implantarlos en el programa de la siguiente forma:

- N1_Local. El selector del tablero de la sala de control de la bahía Jamondino 1 se debe encontrar en posición Local.

- Int_Remoto. El estado del selector en el tablero de patio se debe encontrar en remoto.

- Selector_Sal_Norm. La posición del selector del tablero Jamondino 1 que se encuentra en la sala de control debe estar en Normal.

- Cerrado_Int. El interruptor se debe encontrar en la posición cerrado.

De todos los enclavamientos que se utilizaron en el programa del Quantum, en el programa del PLC Twido (Telemecanique, 2007), se utilizaron los enclavamientos básicos para proporcionar seguridad al sistema. Este sistema de redundancia inicia su proceso cuando ocurra alguna falla en PLC principal. La lógica de los enclavamientos que se realizó en el sistema es más segura, ya que en el momento en que haya algún descuido del personal de trabajo, el sistema realiza una lectura por medio de los PLC, sin dejar que el sistema incurra en alguna falla. 


\section{Resultados}

\section{Protocolo de pruebas}

En este ítem se explicará en qué consiste cada prueba realizada a los tableros de Jamondino 1, Jamondino 2 y Yumbo. Los resultados de las

\section{Inspección de gabinetes}

Se realizó con el objetivo de confrontar las características constructivas del gabinete, con las especificaciones técnicas. Se hizo a través de una

\section{Revisión de equipos}

Se realizó con el objetivo de verificar el estado de los equipos y su disposición en el gabinete, de acuerdo al plano de implantación respectivo, con énfasis en la operatividad y en la facilidad de acceso para el cableado interno y externo. Se hizo a través de inspección visual externa de los equipos que conforman el gabinete, en los que se verificó: ubicación del equipo, referencia,

\section{Revisión alambrado fuerza y control}

Se realizó con el objetivo de revisar que el cableado de fuerza y control del gabinete esté de acuerdo con los planos eléctricos respectivos. Se hizo a través de la revisión de continuidad

\section{Revisión de tensiones}

Se realizó con el objetivo de verificar el buen funcionamiento de las fuentes del tablero. Se hizo a través del totalizador, con todas las protecciones del tablero en posición abierto y las fuentes apagadas. Luego, se revisó que la tensión de la acometida estuviera presente y dentro de lo especificado como tensión nominal (tolerancia pruebas que se hicieron a los equipos del sistema se encuentran a continuación.

inspección visual y medición del gabinete, utilizando como instrumentos: plano con dimensiones del gabinete y flexómetro.

facilidad de acceso y maniobra, identificación y estado. Al mismo tiempo, se hizo una revisión de haces de cables, en cuanto a alineación, ángulos, cruces y amarres. También se revisó el espacio disponible en canaletas, para el cableado externo, y la accesibilidad de las borneras, haciendo uso de instrumentos tales como planos de implantación y listado de materiales.

punto a punto, siguiendo los planos eléctricos, conocida también como "timbrado". Para ello se usaron multímetro y planos eléctricos.

10\%). Se cerró el totalizador y se comprobó que las tensiones se distribuyeran de acuerdo a planos eléctricos. A continuación, se energizaron fuentes para verificar tensiones de salida. Finalmente, se cerraron una a una las protecciones, verificando la presencia de tensión aguas abajo. Se utilizaron multímetro y planos eléctricos. 


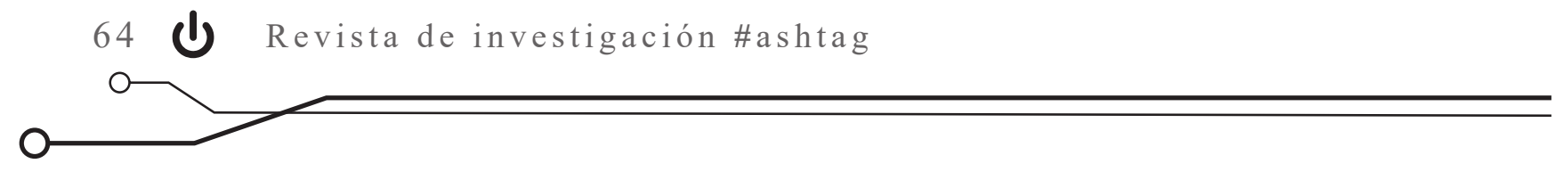

\section{Revisión de energización de equipos}

Se realizó con el objetivo de verificar que cada equipo, al ser energizado, presentara indicación luminosa de funcionamiento correcto. Ello se realizó de la siguiente manera. Una vez energizado el gabinete, se colocaron las protecciones

del controlador, del medidor multifuncional, del interfaz humano-máquina y todos los elementos electromecánicos en posición cerrado. Se usó: listado de materiales, diagramas de localización y planos eléctricos.

\section{Pruebas de los componentes del programa}

Para realizar las pruebas de los componentes del programa, se ingresó al software Vijeo Designer. Luego se abrió el proyecto Jamondino 1, que se estaba programando. Ahí aparecieron todos los paneles que se crearon, en los que se encontró el ítem "saber", al que se le dio clic derecho, lo que desprendió una lista con varias opciones, entre las cuales apareció la función nombrada como "iniciar simulación de dispositivo". Se ingresó al programa con uno de los tres niveles de

seguridad creados, "visualización, operación o ingeniería".

En uno de los paneles de diagramas unifilares, se activó la señal "cerrar interruptor L140" (ver figura 8). Se dio doble clic en la señal que se quería activar, luego en el icono que representaba la señal, y este cumplió su función. Además. se visualizó en la simulación del dispositivo, al arrojar un valor de 1 .

Figura 18. Pruebas de los componentes del programa.

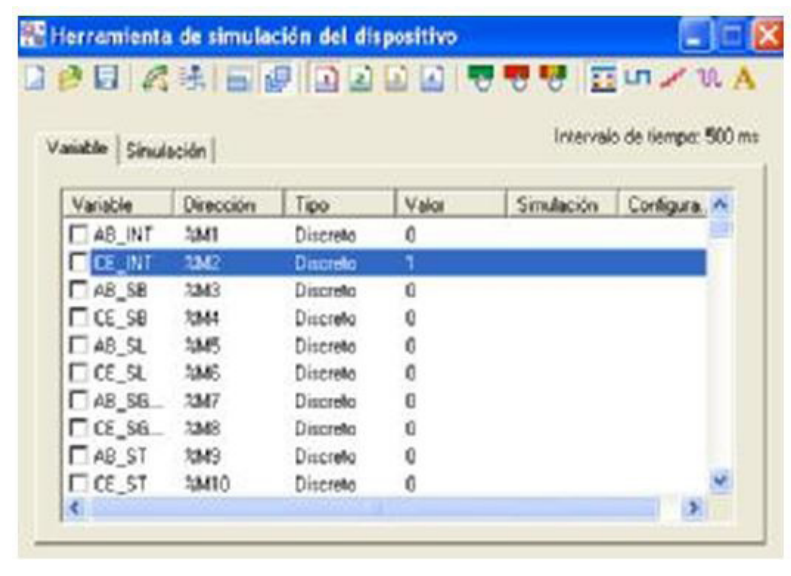

Fuente: elaboración propia.

\section{Planos eléctricos y mecánicos de los tableros de las tres bahías}

El diseño de los planos eléctricos y mecánicos es fundamental para la comprensión del funcionamiento de una bahía, puesto que ambos suministran información importante. Por un lado, los planos eléctricos son herramientas necesarias para identificar en dónde se encuentra 
la alimentación, protección e interconexión de los equipos; por otro, los planos de equipos permiten saber la ubicación exacta de cada equipo, en caso de tener que realizar un cambio.
El diseño de los planos eléctricos y mecánicos de las bahías se realizó teniendo en cuenta el dimensionamiento de los tableros y la cantidad de señales de cada bahía, es decir, la arquitectura de cada bahía. (ver figura 19).

Figura 19. Arquitectura de cada bahía.

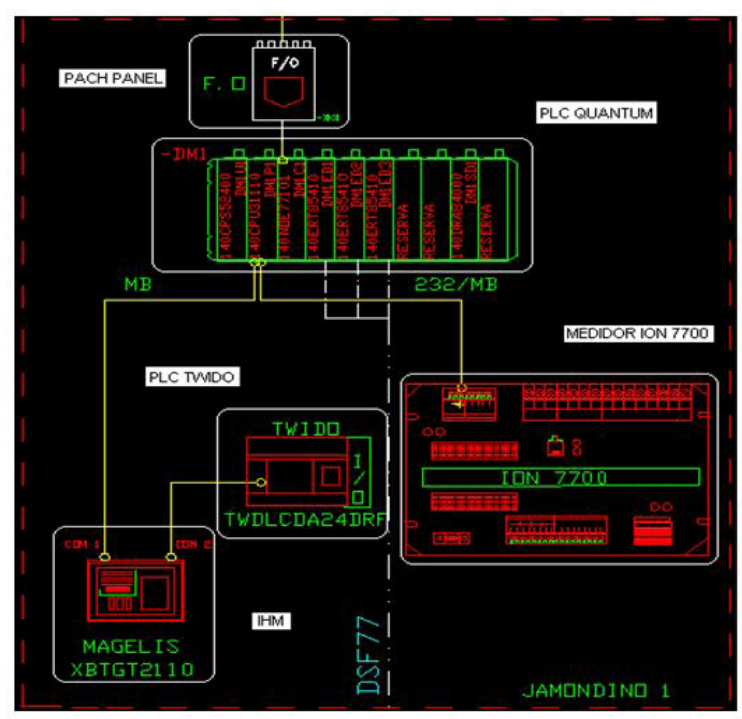

Fuente: elaboración propia.

\section{Conclusiones}

El estado anterior de los equipos de la subestación de San Bernardino era deficiente, como en el caso de los relés, a pesar de que se les había brindado un programa de mantenimiento; lo que había ayudado a que se mantuvieran en operación. Tecnológicamente, sus características no permitían tener un sistema confiable y seguro, pues los equipos eran antiguos, razón por la cual se diseñó e implementó el sistema de automatización de esta subestación.

Por medio de la implementación de este proyecto, se disminuyeron las fallas en la Subestación San
Bernardino, lo que mejoró la calidad del servicio. Se espera que ello se vea reflejado en beneficios económicos para ISA.

Los criterios de automatización (Solano, 2013) realizados en este proyecto permitieron a la subestación tener una integración que proporcionará beneficios en mejoramiento de la funcionalidad, mantenimiento y fiabilidad.

Con el sistema de automatización de la subestación San Bernardino, se obtuvieron los siguientes beneficios: 


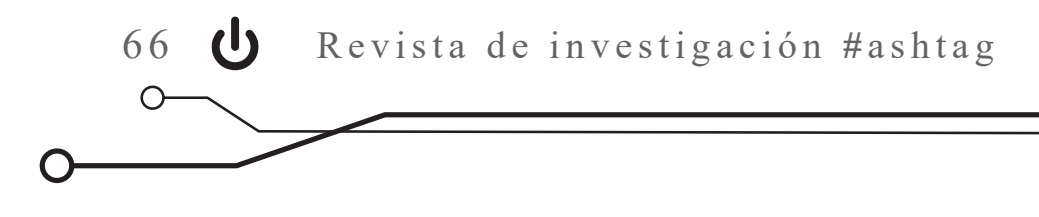

- Restablecimiento más rápido del servicio.

humano-maquina, así como también se controla

- Disminución de costos en operación y mantenimiento.

- Control global del sistema.

- Disponibilidad inmediata de información.

- Aumento de seguridad para el personal.

- Reajuste de las protecciones.

- Mejora de la productividad.

Con la Automatización de la subestación de San Bernardino, se garantizó el registro su funcionamiento desde el centro de control. Se recomienda realizar programas de mantenimiento con más frecuencia a los equipos de la subestación, para prevenir problemas en los equipos instalados.

El ajuste de las protecciones está determinado por la capacidad y el comportamiento de los equipos e instalaciones del sistema eléctrico, por lo que se deben considerar todas las condiciones de operación tanto temporales como permanentes.

\section{Referencias}

Andreula, L. (2010). Red de monitoreo y supervisión de las variables eléctricas en subestaciones. Recuperado de: https:/ / bit.ly/3sl4SBi

Chuncho, J. C. (2013). Modernización y automatización de la subestación Gonzanamá perteneciente al sistema eléctrico de potencia par la Empresa Eléctrica Regional del Sur SA (Loja) (Trabajo de grado, Universidad Politécnica Salesiana) . Recuperado de: http:/ / bit.ly/2P0wWLX

Salazar, V. D. (2015). Propuesta de automatización de una subestación eléctrica utilizando el protocolo IEC-61850 (Trabajo de grado, Universidad de San Carlos de Guatemala). Recuperado de: http:// bit.ly/3umNNJ9

Schneider electric ${ }^{\circledR}$. (2009). Quantum Unity CPU 140CPU31110 [manual de usuario]. Recuperado de: https://bit.ly/2OZZUeV

Schneider electric ${ }^{\circledR}$. (2014). Modicon Quantum with unity Ethernet Network Modules [manual de usuario]. Recuperado de: https:/ / bit.ly/2NTdC2R

Solano, C. D. (2013). Estudio de factibilidad para la implementación del sistema de automatización de subestaciones en la EmelNorte mediante tecnología inteligente (Trabajo de grado, Universidad Politécnica Salesiana). Recuperado de: http://bit.ly/3sjoqGj 
Telemecanique, (2007a). Manual de funcionamiento de TwidoSoft [manual de usuario]. Recuperado de: https:/ / bit.ly/3kduey7

Telemecanique (2007b). Manual del usuario de Magelis XBT GT [manual de usuario]. Recuperado de: https:/ / bit.ly/3pJPIEa

Toscano, M. A. (2010). Automatización de una Subestación Eléctrica utilizando el Protocolo IEC 61850 y el ICCP para el envio de Datos (Trabajo de grado, Universidad Ricardo Palma). Recuperado de: http://bit.ly/3aJPsAU 